\title{
FXR Fast Beam Imaging Diagnostics
}

\author{
M. Ong \\ T. Ferriera \\ R. Gilliam \\ J. Zentler \\ P. Wargo
}

This paper was prepared for submittal to the 12th International Pulsed Power Conference 1999

Monterey, CA

June 27-30, 1999

June 1999

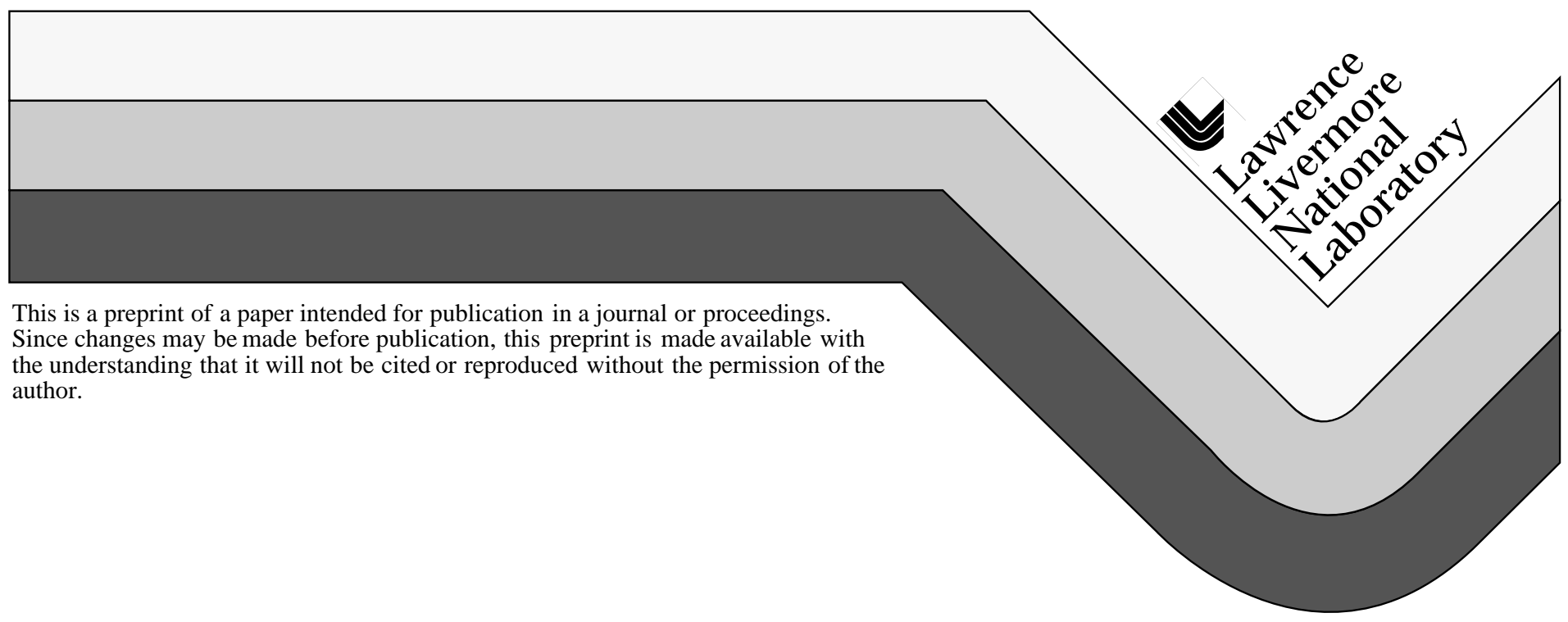




\section{DISCLAIMER}

This document was prepared as an account of work sponsored by an agency of the United States Government. Neither the United States Government nor the University of California nor any of their employees, makes any warranty, express or implied, or assumes any legal liability or responsibility for the accuracy, completeness, or usefulness of any information, apparatus, product, or process

disclosed, or represents that its use would not infringe privately owned rights. Reference herein to any specific commercial product, process, or service by trade name, trademark, manufacturer, or otherwise, does not necessarily constitute or imply its endorsement, recommendation, or favoring by the United States Government or the University of California. The views and opinions of authors expressed herein do not necessarily state or reflect those of the United States Government or the University of California, and shall not be used for advertising or product endorsement purposes. 


\title{
FXR FAST BEAM IMAGING DIAGNOSTICS
}

\author{
M. Ong, T. Ferriera, R. Gilliam, J. Zentler, P. Wargo* \\ Lawrence Livermore National Laboratory, 7000 East Avenue, L-153 \\ Livermore, California
}

\begin{abstract}
The Lawrence Livermore National Laboratory Flash $\mathrm{X}$-ray (FXR) machine is being upgraded to produce two pulses. A very fast imaging system has been developed to characterize the electron beam diameter and shape. The system consists of a kapton target insertion mechanism and a framing camera. It has a fast gated imaging tube (500 ps) and CCD subsystem to capture and send the image to the control room. The beam diameter data provides insight on mechanisms that effect the $\mathrm{x}$-ray spot size. These colorful beam measurements will be compared with our other diagnostics to form a more complete picture of beam behavior. A demonstration will be described where the image data was used to design a collimator to improve $\mathrm{x}$-ray beam performance.
\end{abstract}

\section{INTRODUCTION}

Lawrence Livermore National Laboratory's Flash $\mathrm{X}$-Ray machine (FXR) is a tool to radiograph various assemblies. FXR is an induction linear accelerator. An injector introduces an electron beam into the FXR accelerator. (See Figure 1.) After passing through the accelerator, the beam enters a drift section that directs it toward a thin strip of tantalum, called a target. As the high-energy electrons pass through the target, the electric field created by the stationary charged particles of the heavy tantalum nuclei causes the electrons to decelerate and radiate some of their energy in the form of X-rays. A recently completed upgrade to the FXR improved the quality of the beam. LLNL is currently completing a double-pulse feature to the FXR to provide two separate radiographs.

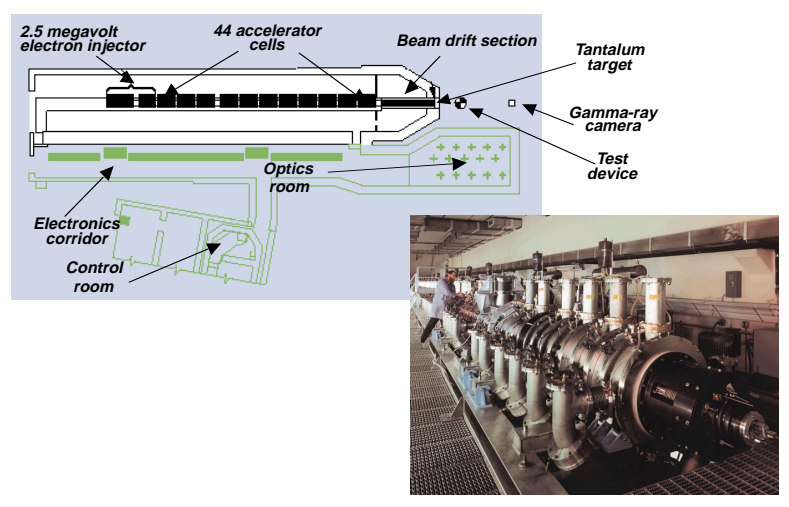

Figure 1. LLNL Flash X-ray (FXR) linear induction accelerator.

When the FXR machine was first turned on in the 1980s, it did not have optical diagnostics for characterizing the electron beam. Within the last few years, three different optical diagnostics were added. The goal was to determine at least the electron beam radius. (1) A modest speed-framing camera with shutter times of more than $10 \mathrm{~ns}$ recorded images that varied from shot-toshot. Beam motion likely created the variations. (2) A streak camera generated images that included both beam motion and beam radius. However, it was difficult to deconvolve the motion from the radius measurement. (3) A couple of years ago a fast framing camera was developed. It produced images that were very consistent and provide information that was used to demonstrate the possibility of improving the quality of the beam. This paper describes the fast electron beam diagnostics and its application to improving the beam.

\section{CAMERA DESIGN}

The accelerator design and tuning teams needed data so they could reduce the x-ray spot size and improve beam transport. The beam characteristics that had to be measured were beam radius and current density. Other beam qualities such as beam divergence and emittance are also important but they would be measured later. Beam dynamics and position also put demands on the camera. These beam characteristics and the resulting camera performance requirements are summarized in Table 1.

\footnotetext{
* Bechtel Nevada Corporation
} 
Table 1. Beam characteristics determined the camera performance requirements

\begin{tabular}{|cl|}
\hline Electron beam characterist ics & Camera Requi rements \\
- Beam radius $(\approx \mathbf{c m})$ & - spatial resolutio $\quad \mathbf{n}(.3 \mathrm{~mm})$ \\
- Beam current de $\mathrm{nsity}$ & - intensity linearity $\quad(\geq 8 \mathrm{bits})$ \\
- Beam motion - BBU $(\approx \mathbf{~ G H z})$ & - shutter speed $\quad(<1 \mathrm{~ns})$ \\
- Beam motion - Corksc rew & - window size \\
- Beam position in pipe & - window size $(\geq 6 \mathrm{~cm})$ \\
\hline
\end{tabular}

There was also a set of operational requirements that drove the design of the camera system. The imaging target would have to be placed in the beam path. The electron to photo converter would perturb the electron beam down stream. Therefore the target had to be retraceable without breaking the beam pipe vacuum and not impact normal machine operation. The beam radius needed to be measured throughout the machine. The only space available for inserting the target in the accelerator section was in the accelerating gap. Fortunately, the drift section near the $\mathrm{x}$-ray target already had an optical diagnostic cross. A target could be conveniently inserted there. In the past, aligning the camera to the target using mirrors was time consuming. In the new design, alignment time had to be minimized. The camera must survive and operate in a high $\mathrm{x}$-ray and radio frequency (RF) noise environment. These operational requirements and the resulting system design requirements are listed in Table 2.

Table 2. Operational requirements determined the camera system design.

\begin{tabular}{|ll|}
\hline -Operational Requirements & -System Design Requirements \\
& \\
-minimize impact on beam transport & -retraceable target \\
-use in accelerator and drift & -insert target in accelerator gap and \\
& diagnostic cross w/o breaking \\
& vacuum \\
-remote operation & -data control links to control room \\
-robust optical alignment & -minimize alignment of mirrors and \\
& target \\
-survive high beam current & -suitable high-temperature target \\
-survive high dose and RF noise & -shielding \\
\hline
\end{tabular}

The design of the fast imaging system is described by the block diagram in Figure 2. Starting at the target, two different target insertion mechanisms were designed. The one for insertion in the acceleration gap was more complex. The diagnostic cross allowed a simple design with the target at $45^{\circ}$ to the beam and direct viewing of the image. The target image is focused onto a coherent fiber optic bundle which conveys photons to a fast gated imager. It serves as the shutter for the camera system and could operate as fast as 500 ps. A CCD camera captures the resulting picture and moves it to the control room for display and storage. By delaying the opening of the shutter, we could capture different sections of the beam. The camera electronics is protected from the $\mathrm{x}$-ray by a RF tight box and lead. Each imaging component will be described.

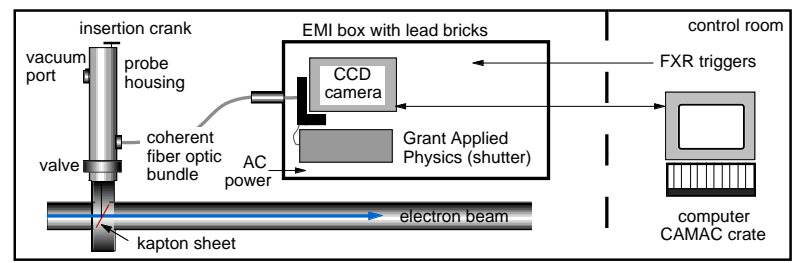

Figure 2. Block diagram of the fast beam imaging system.

The imaging target must intercept a small portion of the $3 \mathrm{kA}$ beam. Kapton with a thickness of $0.005^{\prime \prime}$ was chosen as the target converter material. It can operate at high temperatures $\left(>400^{\circ} \mathrm{C}\right.$ ) and is compatible with the vacuum system. Our initial measurements would focus on obtaining the beam radius, therefore the angular information provided by the transition radiation would not be needed. In order to minimize setup time with target and relay mirror alignment, the smooth finish on the front face was made rough with fine sand paper. This reduced changes in sensitivity with respect to viewing angle.

The insertion probe for the accelerator gap was difficult to design because of the convoluted path. Figure 3 a shows the three mirrors required to relay the image to the fiber bundle. The accelerating gap is not energized when the probe is inserted. The target and mirrors must be fully retracted from the gap when the FXR machine is operating. Figure $3 \mathrm{~b}$ shows a cut-away photograph of the cell with the kapton probe fully deployed. The target must wiggle through the convoluted path to get into position, and we call it the wiggle probe. Figure $3 \mathrm{c}$ shows the insertion mechanism mounted on the FXR with the optical bundle on the lower right. A large diameter vacuum valve allows us to remove the probe mechanism with losing the vacuum in the beam pipe. This design minimized impact on the operation of the FXR machine. Lens packages on both ends of the optical bundle focus the image for the camera.

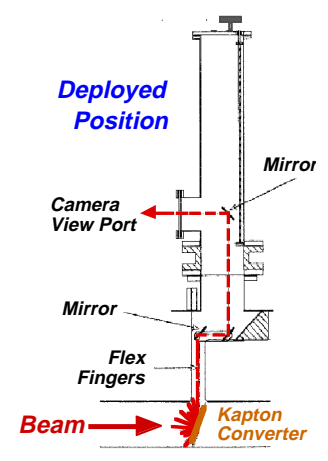

Figure 3a. The insertion mechanism for the accelerator gap has three mirrors. 


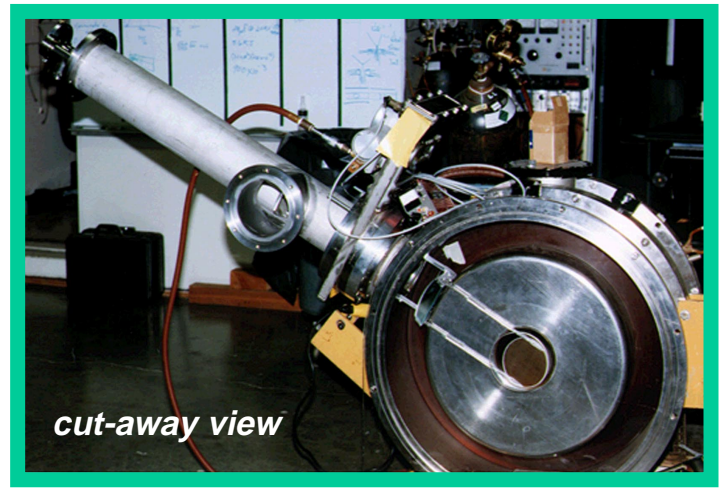

Figure 3b. Cut-away of the cell shows the kapton target fully deployed.

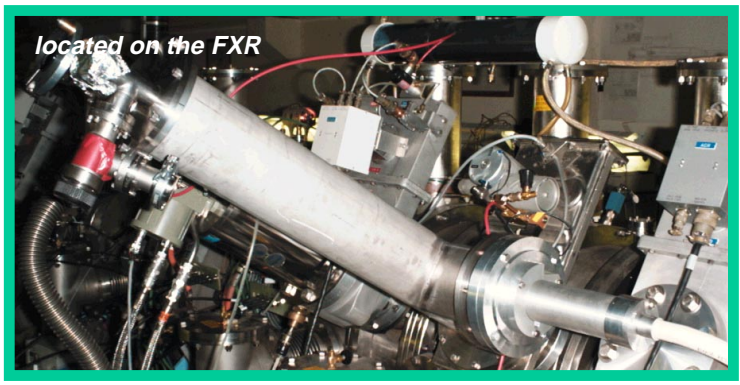

Figure 3c. The insertion mechanism minimizes impact on the operation of the FXR machine.

The camera electronics consists of a Grant Applied Physics gated imager, a computer controlled CCD camera and display, and computer controlled Stanford delay unit. 
The hardware is shown in Figure 4. The gated imager acts as the shutter and the speed can be set as low as 500 ns. This is sufficiently fast to stop beam motion caused by beam breakup instability (BBU) which has a major component at $820 \mathrm{MHz}$. The image in the figure was taken with the wiggle probe. It elliptical shape was caused by the steep angle of the target with respect to the beam path. An LLNL software package, Mistro, supports simple image processing. This includes pseudo-coloring, background subtraction, and line-out reading of current density.

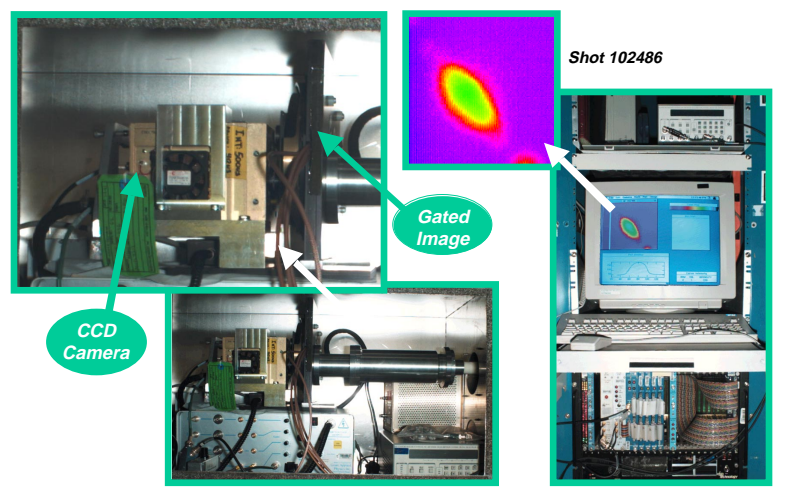

Figure 4. Electronic components of the fast imaging system.

\section{Improving Beam Quality}

Last year, a series of images was taken in the drift section. (See Figure 5.) The shutter delay increased by $10 \mathrm{~ns}$ increments. Two shots were taken at each beam position. The pictures showed a very consistent beam. The beam diameter was about $1 \mathrm{~cm}$, as predicted. It was calculated by averaging the full-width half-maximum of the amplitude in both $\mathrm{x}$ and $\mathrm{y}$ directions and over two shots. The linearity was checked. The beam density integrated over the beam area should be equal to the beam current. The linearity was acceptable. However, the electron beam asymmetry was a surprise. BBU was well controlled but there may be some slower beam centroid motion. Beam spread appeared to be Gaussian and further analysis is needed. The source of the asymmetry will be investigated at a later time. The path we chose to improve $\mathrm{X}$-ray performance was to construct a collimator in the draft section at the end of the accelerator.

The goal of collimation is to improve the figure-ofmerit (FOM) of the $\mathrm{x}$-ray beam. LLNL defines this to be dose divided by spot size squared. While collimation would reduce the dose, the hope was to reduce the stop size. The electron beam quality could be improved in three possible ways. (1) The low-energy electrons in the head and tail of the beam could be scraped off if they could be made to move in a large radius. (2) Some beam motions in the main beam could be eliminated if the collimator diameter was just right. (3) We could reduce the beam spread, especially from the asymmetry, with the right size hole.

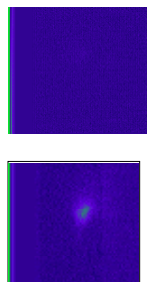

$10 \mathrm{~ns}$

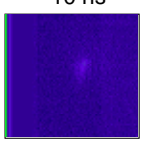

0 ns

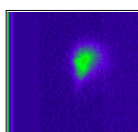

20 ns

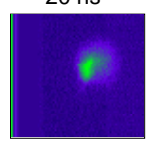

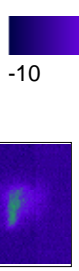

30 ns

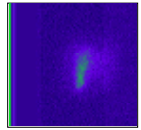

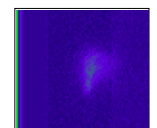

$40 \mathrm{~ns}$

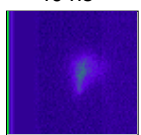

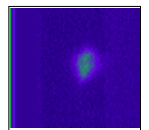

$50 \mathrm{~ns}$

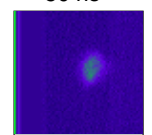

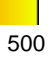

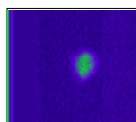

$60 \mathrm{~ns}$

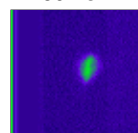

$90 \mathrm{~ns}$
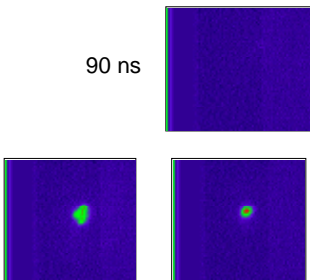

$70 \mathrm{~ns}$

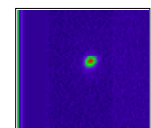

80 ns
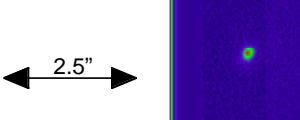

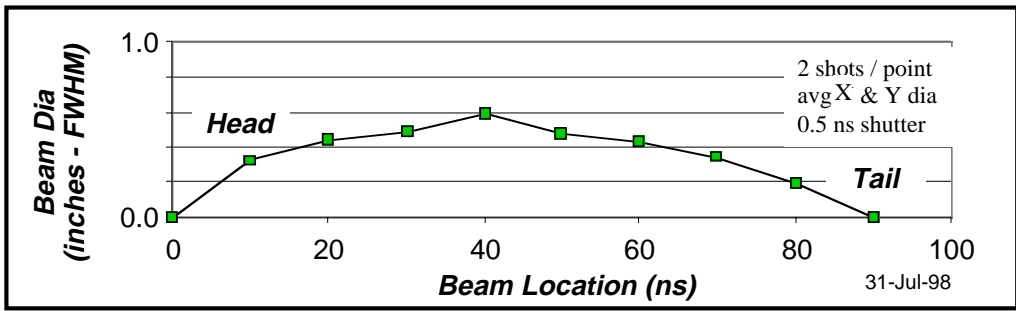

Figure 5. Images taken in the drift section show asymmetric beam cross sections. 
We started with a collimation hole that allowed about $70 \%$ of the beam to pass. Larger diameters were also tried. The result was the FOM for the x-ray beam went from 100 to 125 . Figure 6 . shows the data collected from two other types of sensors, beam bugs and B-dots. They show that while current is reduced, both slow and fast beam motions appear to be greatly reduced. There are still some problems that must be corrected. Nonetheless, we demonstrated the value of collimation. There are of course other ways to improve the FOM by adjusting and improving the FXR machine. These are being pursued.

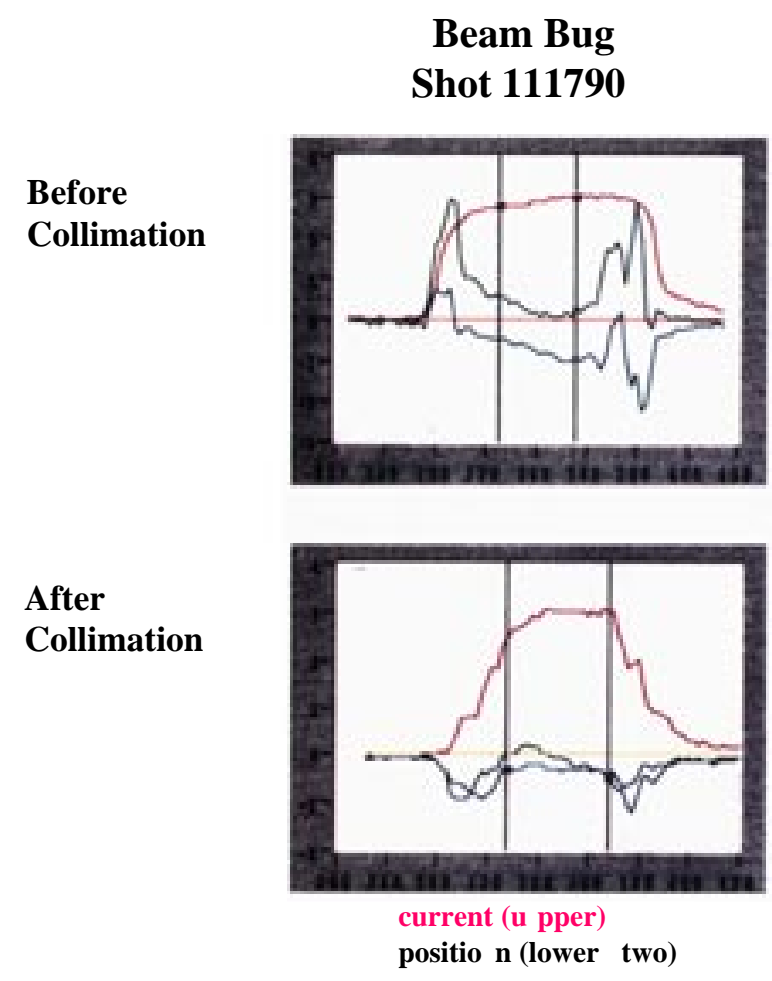

\section{B-dot \\ Shot 111722 and 790}
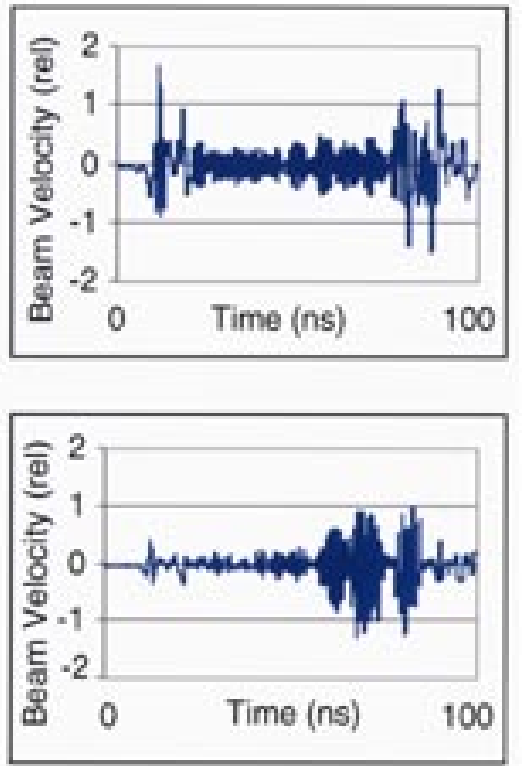

Figure 6. Beam bug and B-dot sensors report reduced beam motion due to collimation.

\section{Future Efforts}

A number of tasks have been proposed. They include identifying a shade created by the collimation, getting more beam radius data throughout the FXR machine, and determining beam divergence with a second target and camera. This will aid beam transport modeling and tuning. If the kapton target was replaced with a fast scintillating material and placed in the path of the x-ray beam, its radius could be recorded for electron beam and $\mathrm{x}$-ray target studies. We believe that fast optical diagnostics is an important diagnostic tool for accelerator development and tuning.
This work was performed under the auspices of the U.S. Department of Energy by Lawrence Livermore National Laboratory under Contract W-7405-Eng-48. 